\title{
Short knee radiographs can be inadequate for estimating TKA alignment in knees with bowing
}

\author{
Sung-Mok Oh${ }^{1}$, Seong-II Bin ${ }^{2 *}$, Jae-Young Kim², Bum-Sik Lee² and Jong-Min Kim²
}

\begin{abstract}
Purpose: The aim of this study was to compare the discrepancy of alignment categorization in total knee arthroplasty (TKA) between the anatomical femorotibial angle (aFTA) measured on short knee radiographs and the mechanical hipknee-ankle axis angle (mHKA) measured on full-length radiographs in knees with and without bowing.

Methods: From January 2014 to June 2017, 107 of 526 osteoarthritic knees at our hospital were found to have femoral or tibial bowing. Bowing was defined as a femoral bowing angle (FBA) $>3^{\circ}$ or $<-3^{\circ}$ or a tibial bowing angle (TBA) $>2^{\circ}$ or $<-2^{\circ}$ in full-length preoperative radiographs. Among 419 knees without bowing, we selected 107 knees as a control group using propensity-score matching. Postoperative alignments were categorized by aFTA in short knee radiographs and $\mathrm{mHKA}$ in full-length radiographs into neutral $\left(2^{\circ} \leq \mathrm{aFTA} \leq 7^{\circ},-3^{\circ} \leq \mathrm{mHKA} \leq 3^{\circ}\right)$, varus $\left(\mathrm{aFTA}<2^{\circ}, \mathrm{mHKA}<\right.$ $-3^{\circ}$ ), and valgus (aFTA $>7^{\circ}, \mathrm{mHKA}>3^{\circ}$ ) alignments. We compared the categorization of alignments between knees with and without bowing using the McNemar test and used logistic regression to find factors for the alignment discordance.
\end{abstract}

Results: Coronal alignment was discordant in $26.2 \%$ of the knees with bowing and $13.1 \%$ of the knees without bowing $(p<0.001)$. FBAs were a significant factor affecting the discordance of alignment categorization $(\mathrm{OR}=1.152,95 \% \mathrm{Cl} 1.038-$ $1.279, p=0.008)$.

Conclusion: Short knee radiographs are insufficient for estimating coronal alignment after TKA, particularly in knees with femoral bowing.

Level of evidence: IIl: Retrospective comparative study.

Keywords: Knee, Arthroplasty, Alignment, Bowing, Short radiograph, Full-length radiograph

\section{Introduction}

Postoperative coronal alignment is an important factor in determining the longevity of a total knee arthroplasty (TKA) procedure [1-8]. The mechanical hip-knee-ankle axis angle (mHKA) measured from full-length radiographs is the gold standard for estimating coronal alignment [9]. However, this measure requires special equipment, generates extra costs, and irradiates the pelvic organs [10]. For these reasons, the anatomical femorotibial angle (aFTA) measured from short knee radiographs is often used in clinical studies to estimate

\footnotetext{
*Correspondence: sibin@amc.seoul.kr

${ }^{2}$ Department of Orthopedic Surgery, Asan Medical Center, University of Ulsan College of Medicine, 88, Olympic-ro 43-gil, Songpa-gu, Seoul 05505, Republic of Korea

Full list of author information is available at the end of the article
}

the alignment of TKAs $[1,6,7]$. The aFTA is used in many knee-scoring systems, including the modified Hospital for Special Surgery (HSS) knee score [11], the Knee Society Score (KSS), and even the revised KSS [12].

Interestingly, studies that have used short knee radiographs and those that utilized full-length radiographs to determine the longevity of TKAs in accordance with the postoperative alignment have reported different results. The former have reported that a neutral alignment produces a superior longevity than outliers $[1,6,7]$. The latter studies have indicated no differences between neutral alignment and outliers in terms of TKA longevity [2, 3, 8,13 . Notably however, although many studies of TKAs have been performed using short knee radiographs to evaluate postoperative alignment $[1,6,7,14]$, the

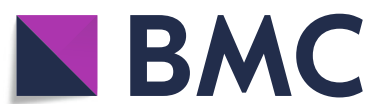

Part of Springer Nature (c) The Author(s). 2020 Open Access This article is distributed under the terms of the Creative Commons Attribution 4.0 International License (http://creativecommons.org/licenses/by/4.0/), which permits unrestricted use, distribution, and reproduction in any medium, provided you give appropriate credit to the original author(s) and the source, provide a link to the Creative Commons license, and indicate if changes were made. The Creative Commons Public Domain Dedication waiver (http://creativecommons.org/publicdomain/zero/1.0/) applies to the data made available in this article, unless otherwise stated. 
question of whether short knee radiographs can replace full-length radiographs remains disputed. Some previous reports have found a small difference of less than $1^{\circ}$ between the aFTA on short knee radiographs and mHKA on full-length radiographs and a high correlation $(r=$ $0.8)[15,16]$. By contrast, other investigations have reported a large difference of up to $7.2^{\circ}$ and a low correlation $(r=0.34-0.41)$ between the aFTA and mHKA [10, 17-19]. The reported alignment categories of neutral, varus, and valgus for the aFTA and mHKA are discordant over a variable range (15-70\%) [17, 20-22].

Extra-articular deformities, such as femoral and tibial bowing, could have caused such discrepancies in the results of previous studies [17, 20-22]. To date however, no studies have reported on the percentage of neutral alignments of the aFTA measured from short knee radiographs that would also be neutral alignments of the mHKA on a full-length radiograph in knees with or without bowing. Hence, we performed a direct comparison of the discordance in alignment categorization between knees with and without bowing in our present study. We speculated that these analyses would provide meaningful data that indicated why previous studies showed different results.

We hypothesized that coronal bowing itself could cause aFTA categorizations measured from short radiographs to be less accurate than the corresponding mHKA categorizations measured from full-length radiographs in patients who have undergone TKA. We thus investigated the prevalence of discordance between these two sets of categorizations in accordance with the existence of femoral or tibial bowing.

\section{Materials and methods}

Preoperative and postoperative radiographs of 485 patients (bilateral 215, unilateral 270) who underwent a TKA at our hospital between January 2014 and June 2017 at our hospital were retrospectively reviewed. Among the 700 knees of these 485 consecutive cases, we applied the following exclusion criteria: diagnosis other than primary osteoarthritis (e.g., rheumatoid arthritis, osteonecrosis, or traumatic osteoarthritis), flexion contracture of over $10^{\circ}$, previous history of operation on the ipsilateral limb (e.g., hip arthroplasty, internal fixation of a femoral or tibial fracture, unicompartmental knee arthroplasty, high tibial osteotomy), or inadequate rotation on radiographs. In all, 526 knees of 380 patients were screened in our current study. Among these 526 knees, 107 were identified with femoral or tibial coronal bowing in preoperative weight-bearing full-length radiographs. We matched 107 knees without bowing using age, sex, side, and preoperative flexion contracture in accordance with the propensity score using $\mathrm{R}$ software. A total of 214 knees of 182 patients (107 each in the bowing and no bowing groups) were therefore enrolled in the final study cohort (Fig. 1).

\section{Preoperative radiographs}

The femoral bowing angle (FBA) and tibial bowing angle (TBA) were determined from preoperative standardized full-length hip-knee-ankle weight-bearing anteroposterior (AP) radiographs, measuring $14 \times 51$ in.. Full-length weight-bearing radiographs were obtained with the subjects standing barefoot in a position in which the patella was oriented forward (Fig. 2). Femoral bowing was defined as an FBA $>3^{\circ}$ or $<-3^{\circ}$ [23]. Tibial bowing was defined as a $\mathrm{TBA}>2^{\circ}$ or $<-2^{\circ}[19,24]$. All lateral bowing was recorded as positive, whereas medial bowing was recorded as negative.

\section{Postoperative radiographs}

The aFTA was measured from routine $14 \times 17 \mathrm{in}$. weight-bearing AP radiographs, with the patellae facing forward and the legs in full extension, and the mHKA was measured from routine full-length hip-knee-ankle weight-bearing AP radiographs measuring $14 \times 51 \mathrm{in}$. (Fig. 3). Routine weight-bearing AP and lateral knee radiographs $(14 \times 17$ in. cassette $)$ and full-length standing films $(14 \times 51 \mathrm{in}$. cassette) were taken at 6 weeks, 3 months, 6 months, 1 year, and every $2-3$ years thereafter. Pairs of standing short AP knee radiographs and standing full-length AP radiographs, taken on the same day and in the same position, were evaluated. We measured parameters from radiographs taken within 1.5 years. The alignments on short knee radiographs were classified as neutral $\left(2^{\circ} \leq \mathrm{aFTA} \leq 7^{\circ}\right)$, varus $\left(\mathrm{aFTA}<2^{\circ}\right)$ or valgus $\left(\mathrm{aFTA}>7^{\circ}\right)[1,7,20,25]$. Postoperative mHKA was expressed as a deviation from $0^{\circ}$, with a positive value for the valgus direction and negative value for the varus direction. The alignments determined from full-length radiographs were also classified as neutral $\left(-3^{\circ} \leq \mathrm{mHKA}\right.$ $\left.\leq 3^{\circ}\right)$, varus $\left(\mathrm{mHKA}<-3^{\circ}\right)$, or valgus $\left(\mathrm{mHKA}>3^{\circ}\right)[2,8$, 13] .

To evaluate intra- and inter-observer reliability, 20 knees were randomly selected, and all parameters were measured twice by two observers with a 2 -week interval between measurements. The reliability of the data was expressed in terms of the intraclass correlation coefficient (ICC). The ICC values were over 0.90 in all cases and for all radiographic parameters except for the TBA. Those for the TBA were also greater than 0.85 however. As the reproducibility of all measurements was good to excellent, the parameters measured by only one experienced orthopedic surgeon were used in this study. The measurements were made using the tools from a picture-archiving communication system (PACS). The least detectable angular change was $1^{\circ}$. 


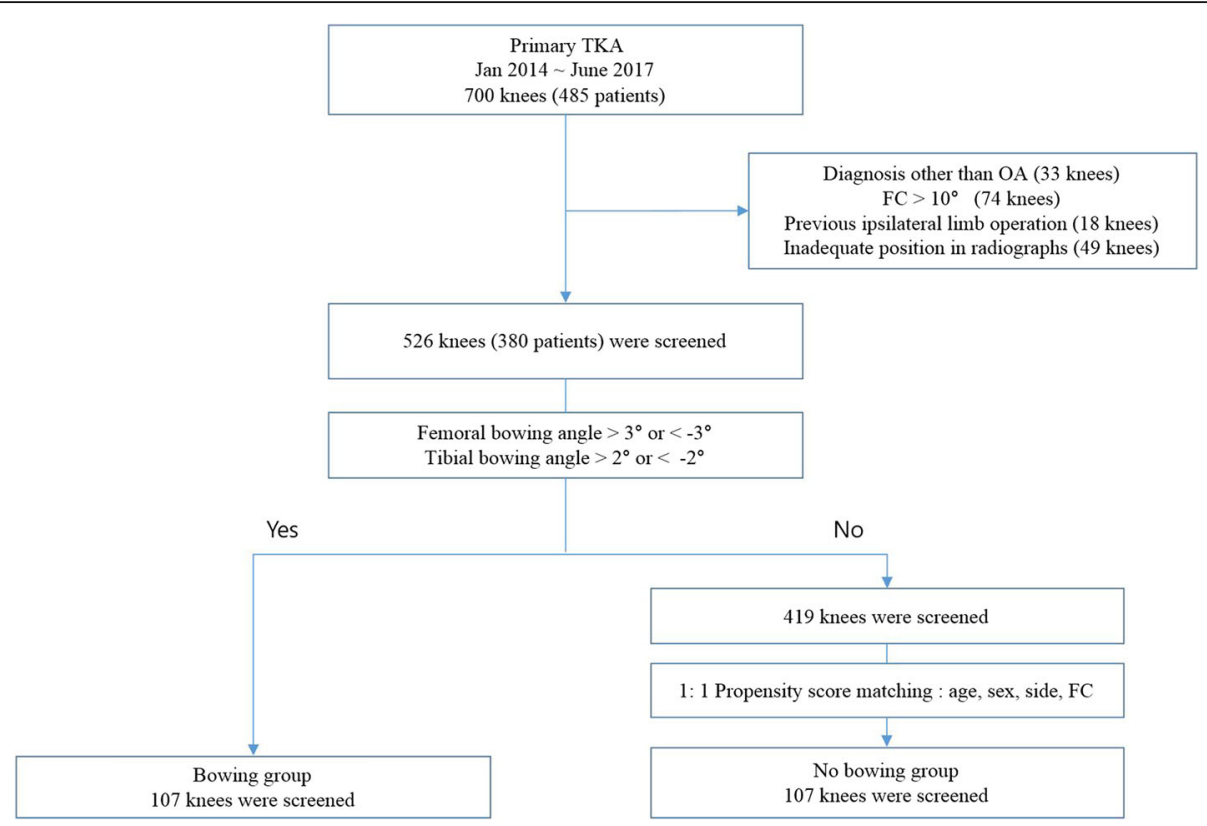

Fig. 1 Study flow diagram. TKA, total knee arthroplasty; OA, osteoarthritis; FC, flexion contracture

\section{Statistical analysis}

The parameters of the study groups, including FBA, TBA, aFTA, and mHKA, are given as means and standard deviations (SDs) (Table 1). Discordance between the aFTA and mHKA with regard to the existence of bowing was investigated using the McNemar test. Comparisons of the differences between the aFTA and mHKA according to knee bowing were analyzed using the MannWhitney $U$ test. The association between bowing and discordance between the categorization of aFTA and mHKA alignment was investigated using univariate logistic regression analyses. The results are presented as odds ratios (OR) and 95\% CIs. All of the statistical analyses other than propensity score matching were carried out using SPSS for Windows version 21.0 (SPSS, Chicago, IL). $P$ values of $<0.05$ were considered significant.

\section{Results}

Knees with femoral bowing made up 15.7\% (83/526) of the total in the initial patient population whereas $8.2 \%$ $(43 / 526)$ of knees had tibial bowing in this initial cohort. Nineteen (3.6\%) of 526 knees had both femoral and tibial bowing. The mean angle differences between the aFTA and $\mathrm{mHKA}$ were $4.74^{\circ} \pm 1.87^{\circ}$ (SD) for the no bowing group and $6.14^{\circ} \pm 2.49^{\circ}(\mathrm{SD})$ for bowing group which was a significant difference $(p<0.001$; Table 1$)$.

In the no bowing study group, $86.0 \%(92 / 107)$ of the TKAs showed a neutral alignment based on the aFTA on short knee radiographs. However, 3.3\% (3/92) of these TKAs that appeared to have a neutral alignment from the aFTA on short knee radiographs were found to have varus or valgus alignments based on the mHKA on full-length radiographs. In the categorization of alignments as neutral, varus, and valgus, 13.1\% (14/107) of our study subjects with no bowing were found to be discordant. In the bowing group, $76.6 \%(82 / 107)$ of the TKAs showed a neutral alignment from the aFTA determination on short knee radiographs. However, $14.6 \%$ $(12 / 82)$ of these TKAs that appeared to have a neutral alignment from the aFTA measurements on short knee radiographs were found to have varus or valgus alignments based on the mHKA on full-length radiographs. With regard to the categorization of neutral, varus, or valgus alignments, $26.2 \%(28 / 107)$ of our study subjects with knee bowing were discordant (Table 2). When comparing the no bowing and bowing groups, the discordance ratio was significantly different $(13.1 \%$ in the no bowing group vs. $26.2 \%$ in the bowing group, $p<$ 0.001; Table 3). The discordance among alignment categorizations was not affected by the degree of TBA ( $p=$ 0.704, OR 1.035, 95\% CI 0.867-1.235), but affected by the degree of FBA $(p=0.008$, OR $1.152,95 \%$ CI $1.038-$ 1.279). For each $1^{\circ}$ increase in the FBA, the odds of discordance were 1.152 times greater. When the FBA increased more than $5^{\circ}$, the odds of discordance was almost doubled.

\section{Discussion}

To the best of our knowledge, our current study is the first to suggest discordance between the alignments in knees with or without bowing that are categorized by the aFTA from short knee radiographs and the mHKA 


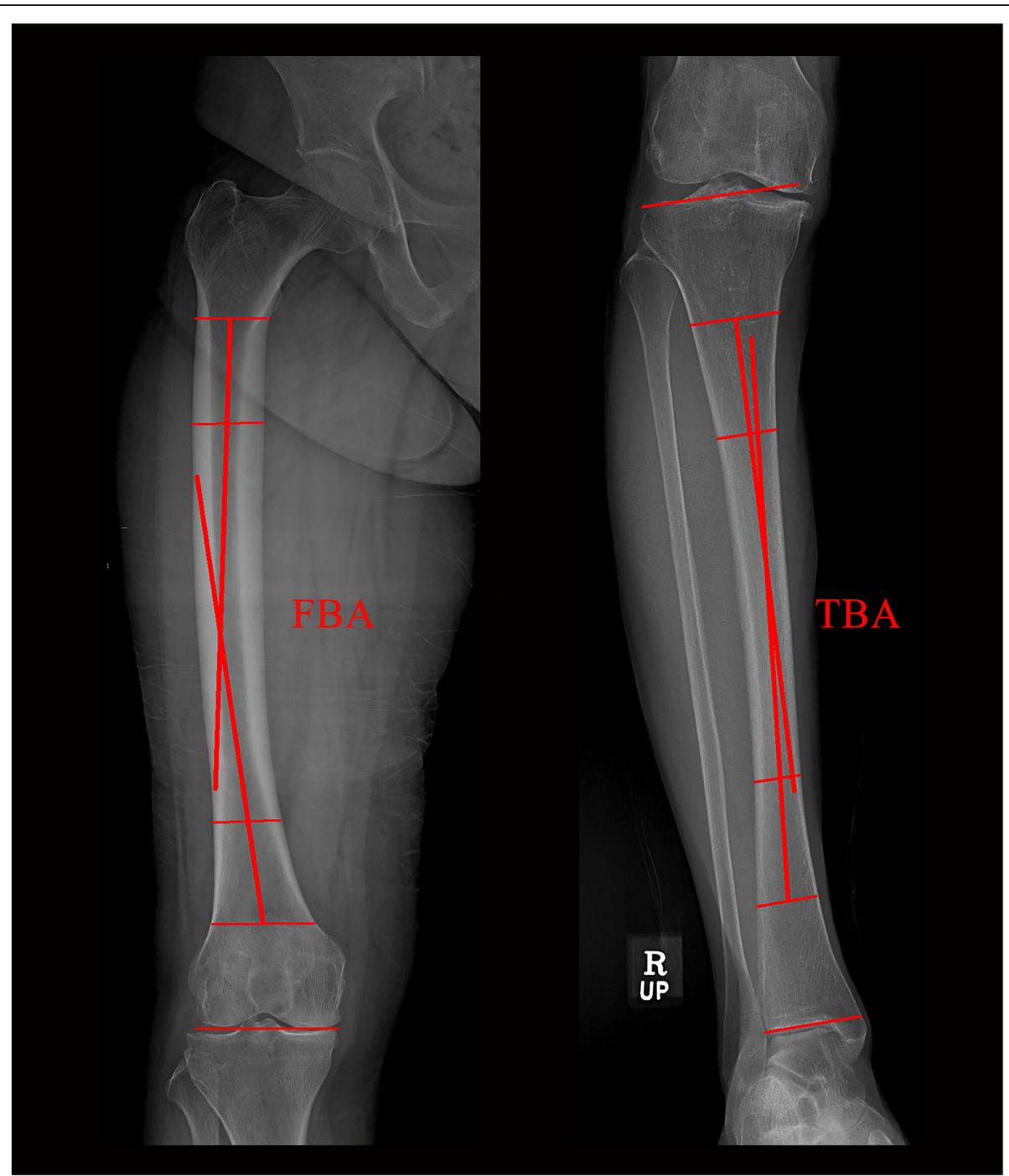

Fig. 2 Preoperative full-length $(14 \times 51$ in. grid cassette) radiograph showing the measurement of the femoral bowing angle (FBA) and tibial bowing angle (TBA). The FBA was defined as the angle between the line connecting the points bisecting the femur at $0 \mathrm{~cm}$ and $5 \mathrm{~cm}$ below the lowest portion of the lesser trochanter and the line connecting the points bisecting the femur at $5 \mathrm{~cm}$ and $10 \mathrm{~cm}$ above the lowest portion of the lateral femoral condyle [23]. The TBA was defined as the angle between the line connecting the points bisecting the tibia at $5 \mathrm{~cm}$ and $10 \mathrm{~cm}$ from the highest portion of the lateral tibial plateau and the line connecting the points bisecting the tibia at $5 \mathrm{~cm}$ and $10 \mathrm{~cm}$ from the lowest portion of the tibial plafond [19].

on full-length radiographs. In knees with bowing, alignment categorizations were discordant at a higher rate $(26.2 \%)$ than in knees without bowing (13.1\%) $(p<$ $0.001)$. Bowing that could not be accessed using short knee radiographs has been suggested previously as the cause of discordance between the aFTA and mHKA [19, 21]. Notably, the incidence of bowing is especially high in Asian patients [24, 26, 27]. If the proportion of bowing is higher in a given patient cohort, there would be a greater risk of overestimating or underestimating the alignment. The direct comparisons of the alignment discordance between knees with and without bowing would help to more clearly elucidate the effects of bowing. The results of our present study strongly support that full- length radiographs should be used to evaluate postoperative TKA alignment in knees with bowing.

Alignment discordance between short knee radiographs and full-length radiographs has been reported at rates ranging from $15 \%$ to $70 \%[17,20-22]$. However, previous studies have not considered discordance according to the existence of bowing. Park et al. [20] reported that $33 \%$ of their subjects had discordant alignment in the aFTA and MHKA on postoperative images (neutral criteria: $2.4^{\circ} \leq \mathrm{aFTA} \leq 7.2^{\circ}, \mathrm{mHKA} \leq \pm 3^{\circ}$ ). AbuRajab et al. [22] reported that only $30 \%$ of categorizations were done correctly with the aFTA (neutral criteria: aFTA $<2^{\circ}$, mHKA $0^{\circ}$ ). Van Raaji et al. [17] reported that $15 \%$ of varus alignment knees determined by 


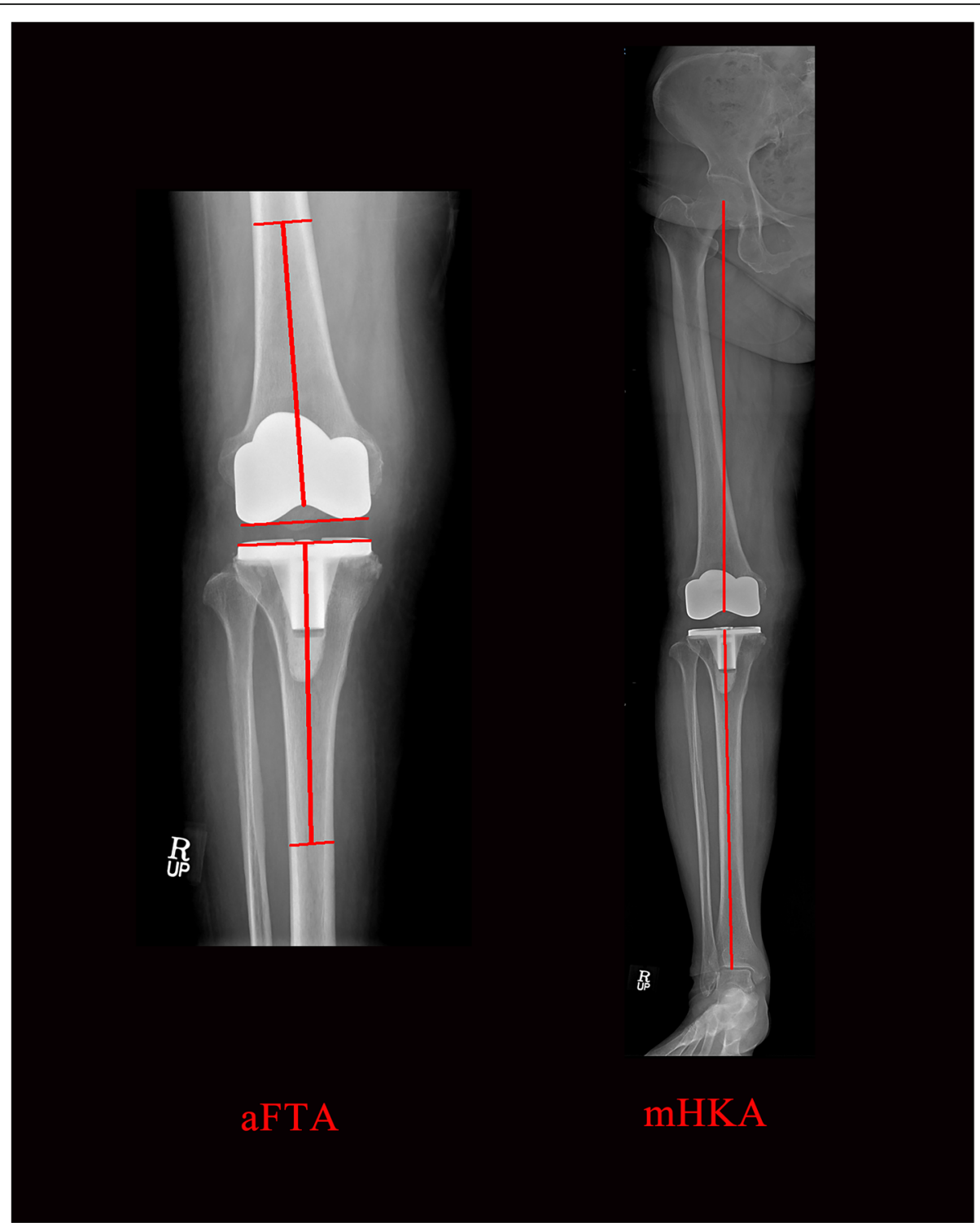

Fig. 3 Postoperative short ( $14 \times 17$ in. grid cassette) and full-length $(14 \times 51$ in. grid cassette) radiographs showing the measurement of anatomical femorotibial angle $(\mathrm{aFTA})$ and mechanical hip-knee-ankle axis angle ( $\mathrm{mHKA})$ The postoperative aFTA was measured using two lines, the first aligned from the bisection point of the femur, $15 \mathrm{~cm}$ above the lowermost portion of the femoral component to the deepest part of the center of the femoral component, and the second from the bisecting point of tibia $15 \mathrm{~cm}$ down from the uppermost portion of the tibial component [19]. Postoperative mHKA was made between the two lines, the first aligned from the center of the femoral head to the deepest part of the center of the femoral component, and the second from the midpoint of the inner extension of the tibio-talar joint to the center of the tibial component [14].

mHKA were classified as having no varus deformity when categorized by aFTA on preoperative radiographs (varus criteria: aFTA $<-2^{\circ}$, mHKA $<0^{\circ}$ ). Lee et al. [21] recorded only a $73 \%$ accuracy between aFTA and $\mathrm{mHKA}$ categorization (neutral criteria: $3^{\circ} \leq \mathrm{aFTA} \leq 9^{\circ}, \mathrm{mHKA} \leq$ $\pm 3^{\circ}$ ). Although there have been variable definitions of neutral alignment $[17,21,22]$, we here confirmed that the discordance of alignment categorization between the aFTA and mHKA in knees without bowing (13.1\%) was lower than the results of previous studies and in the knees with bowing in our present cohort (26.2\%) [17, 20-22].

Our present analyses also demonstrated that the categorization discordance was greater for knees with a greater FBA (OR 1.152, 95\% CI 1.038-1.279, $p=0.008$ ). It has been indicated previously in one study that knees with a greater FBA $(\mathrm{OR}=1.2)$ and $\mathrm{TBA}(\mathrm{OR}=1.2)$ may show a lower accuracy in alignment categorization between aFTA and mHKA [21], and in another report that the FBA and TBA, particularly of the femur, may be 
Table 1 Demographic and radiological data

\begin{tabular}{|c|c|c|c|}
\hline & $\begin{array}{l}\text { No bowing } \\
(n=107)\end{array}$ & $\begin{array}{l}\text { Bowing } \\
(n=107)\end{array}$ & $p$ value \\
\hline Age (years) & $70.01 \pm 5.37$ & $70.10 \pm 5.81$ & $0.968^{a}$ \\
\hline Sex & $\begin{array}{l}\text { M } 3 \\
\text { F } 104\end{array}$ & $\begin{array}{l}\text { M } 5 \\
\text { F } 102\end{array}$ & $0.471^{b}$ \\
\hline Side & $\begin{array}{l}\text { R } 55 \\
\text { L } 52\end{array}$ & $\begin{array}{l}\text { R } 58 \\
\text { L } 49\end{array}$ & $0.681^{b}$ \\
\hline $\mathrm{FC}\left({ }^{\circ}\right)$ & $5.66 \pm 3.46$ & $5.79 \pm 3.71$ & $0.697^{\mathrm{a}}$ \\
\hline Bowing type & none & $\begin{array}{l}\text { Femur only }(n=64) \\
\text { Tibia only }(n=24) \\
\text { Femur \& Tibia }(n=19)\end{array}$ & \\
\hline$|\mathrm{FBA}|\left(^{\circ}\right)$ & $1.08 \pm 1.02$ & $4.65 \pm 2.33$ & $<0.001^{\mathrm{a}}$ \\
\hline $\begin{array}{l}\text { Femoral bowing } \\
\text { direction }\end{array}$ & none & M 14@L 69 & \\
\hline$|\mathrm{TBA}|\left(^{\circ}\right)$ & $0.77 \pm 0.78$ & $1.91 \pm 1.60$ & $<0.001^{\mathrm{a}}$ \\
\hline $\begin{array}{l}\text { Tibial bowing } \\
\text { direction }\end{array}$ & none & M 25@L 18 & \\
\hline aFTA $\left(^{\circ}\right)$ & $3.91 \pm 2.08$ & $5.11 \pm 2.58$ & $<0.001^{\mathrm{a}}$ \\
\hline $\mathrm{mHKA}\left({ }^{\circ}\right)$ & $-0.83 \pm 1.84$ & $-1.04 \pm 2.27$ & $0.379^{a}$ \\
\hline aFTA-mHKA difference $\left(^{\circ}\right)$ & $4.74 \pm 1.87$ & $6.14 \pm 2.49$ & $<0.001^{\mathrm{a}}$ \\
\hline
\end{tabular}

FC Flexion contracture, $|F B A|$ absolute value of femoral bowing angle, $|T B A|$ absolute value of tibial bowing angle, $M$ medial, $L$ lateral, aFTA anatomical femorotibial angle, $M H K A$ mechanical hip-knee-ankle axis angle ${ }^{a}$ Mann-Whitney $U$ test

${ }^{\mathrm{b}}$ Fisher's exact test

major contributors to the divergence between the aFTA and mHKA in TKA patients [19]. Our current findings are compatible with these earlier results in that a greater FBA was found in our present analysis to be a significant factor in the discordance of alignment categorizations. In our current analyses also, $6.0 \%$ of the neutral mHKA cases in the no bowing group and $3.5 \%$ of the patients with this alignment in the bowing group were categorized by aFTA as having a varus alignment on short knee radiographs. However, $14.1 \%$ of those categorized as having a neutral alignment in the mHKA in the bowing group were found to have a valgus alignment in aFTA on short knee radiographs, or nearly triple that of the no bowing group (5.0\%). These results also support the conclusion that there is a tendency for knees with bowing to be measured as having a valgus direction when using short knee radiographs. Femoral bowing usually occurs in the lateral direction and tibial bowing is usually found in the medial direction. Lateral femoral bowing could thus make alignments using the aFTA appear more valgus and medial tibial bowing could make alignments with the aFTA appear more varus than the corresponding alignments determined by the mHKA. The wide range of examples of femoral bowing and relatively small angle and small number of instances of tibia bowing could be the reason for these results.

There were several limitations to our study of note. First, the analysis was retrospective and could be biased in terms of patient selection. However, we reviewed fulllength preoperative radiographs of 700 consecutive TKA cases over an appreciable time period and matched our subject knees with bowing to those without bowing using a propensity score. Second, our sample cohort comprised mainly female Korean subjects undergoing TKA and our results may not therefore be fully applicable to male patients or Western populations. Third, the definition of femoral and tibial bowing and the method used to measure them may have altered the results. In our current analysis, the definition and measurement methods for femoral bowing and tibial bowing were as described in previous studies [19, 23, 24]. Fourth, the definition of neutral alignment could have affected our findings. The range of neutral alignments using the aFTA is reported to be variable $[1,6,12,28]$. Ritter and Fang et al. reported in two studies that included 6070 knees with 7.6 years follow-up and 6070 knees with 6.6 years follow-up, respectively, that an aFTA of between

Table 2 Agreement in the categorization of lower-limb deformity according to the existence of bowing

\begin{tabular}{|c|c|c|c|c|c|}
\hline & & \multicolumn{3}{|c|}{ aFTA in short-leg AP-measured deformity } & \multirow[t]{2}{*}{ Total } \\
\hline & & Varus & Neutral & Valgus & \\
\hline \multicolumn{6}{|l|}{ No bowing group } \\
\hline \multirow[t]{4}{*}{ mHKA on Full-Length AP measured deformity } & Varus & 4 & 2 & 0 & 6 \\
\hline & Neutral & 6 & 89 & 5 & 100 \\
\hline & Valgus & 0 & 1 & 0 & 1 \\
\hline & Total & 10 & 92 & 5 & 107 \\
\hline \multicolumn{6}{|l|}{ Bowing group } \\
\hline \multirow[t]{4}{*}{ mHKA in full-length AP measured deformity } & Varus & 6 & 10 & 1 & 17 \\
\hline & Neutral & 3 & 70 & 12 & 85 \\
\hline & Valgus & 0 & 2 & 3 & 5 \\
\hline & Total & 9 & 82 & 16 & 107 \\
\hline
\end{tabular}


Table 3 Lower-limb deformities in accordance with the existence of bowing

\begin{tabular}{llll}
\hline & Accordance & Discordance & Total \\
\hline No bowing group & $93(86.9 \%)$ & $14(13.1 \%)$ & $107(100 \%)$ \\
Bowing group & $79(73.8 \%)$ & $28(26.2 \%)$ & $107(100 \%)$ \\
\hline McNemar test, $p<0.001$ & & &
\end{tabular}

$2.5^{\circ}$ and $7.4^{\circ}$ measured on short knee radiographs should be considered a neutral coronal alignment $[1,7]$. In most of the previous studies that have used full-length radiographs, the mHKA was considered neutral if it ranged from $-3^{\circ}$ to $3^{\circ}[2,4,8,13]$. Liu et al. reported in their earlier meta-analysis of 10 studies of short knee radiographs and full-length radiographs that an aFTA from $2.5^{\circ}$ to $7.4^{\circ}$ and mHKA from $-3^{\circ}$ to $3^{\circ}$ have been regarded as a neutral alignment up to the present time [5]. Park et al. have also reported the discordance of alignment categorization using same criteria for a neutral aFTA and mHKA without consideration of bowing [20]. As the least detectable angular change was $1^{\circ}$ in our PACS system, we defined a neutral alignment as $2^{\circ} \leq \mathrm{aFTA} \leq 7^{\circ}$ on a short knee radiograph and $-3^{\circ} \leq$ mHKA $\leq 3^{\circ}$ on a full-length radiograph. Fifth, as with all radiographic assessments of coronal plane alignments, lower-extremity rotation and flexion contracture could affect the radiological parameters of full-length radiographs $[29,30]$. However, we used full-length radiographs that were taken using a detailed uniform protocol and we excluded knees with a preoperative flexion contracture of more than $10^{\circ}$. In addition, there was no patient with genu recurvatum who showed a hyperextension of the knee of more than $10^{\circ}$. Sixth, we only analyzed coronal bowing. However, the results from a previous study on femoral bowing indicated a poor correlation between the parameters in the coronal and sagittal planes [31]. Moreover, in other studies on bowing $[19,23,24]$ or reporting on the category of the alignment after TKA [20-22] in the coronal plane, only the parameters measured on films of the coronal plane were used. We speculated that the bowing on the sagittal plane might not significantly affect the coronal alignment of TKAs on radiographs taken in the patella forward position.

\section{Conclusion}

The aFTA measured from short knee radiographs and mHKA measured from full-length radiographs are both used in studies reporting the mid- to long-term outcomes of TKA and in scoring systems to estimate coronal alignment. However, the aFTA measured using short knee radiographs is insufficient for evaluating the coronal alignment after TKA, particularly in knees with femoral bowing. The results of previous studies that have used this approach should therefore be reconsidered, at least in patients with femoral bowing. Full-length radiographs are recommended for analyzing alignment categorizations after TKA.

\section{Abbreviations}

aFTA: anatomical femorotibial angle; FBA: femoral bowing angle; HSS: Hospital for Special Surgery; KSS: Knee Society Score; mHKA: mechanical hip-knee-ankle axis angle; TBA: tibial bowing angle; TKA: total knee arthroplasty

\section{Acknowledgements}

Not applicable.

\section{Authors' contributions}

SM Oh and JY Kim analyzed and interpreted the patient data. SM Oh was a major contributor in writing the manuscript. BS Lee, JM Kim and SI Bin revised manuscript. All authors have read and approved the final manuscript.

\section{Funding}

Not applicable.

\section{Availability of data and materials}

The datasets used and/or analyzed during the current study are available from the corresponding author on reasonable request.

Ethics approval and consent to participate

This study was approved by the Institutional Review Board of Asan Medical Center (Seoul, Korea) (ID number: S2017-1044).

\section{Consent for publication}

Not applicable.

\section{Competing interests}

The authors declare that they have no competing interests.

\section{Author details}

${ }^{1}$ Nanoori Hospital, 156, Jange-ro 156, Bupyung-gu, Incheon 21353, Republic of Korea. ${ }^{2}$ Department of Orthopedic Surgery, Asan Medical Center, University of Ulsan College of Medicine, 88, Olympic-ro 43-gil, Songpa-gu, Seoul 05505, Republic of Korea.

Received: 10 July 2019 Accepted: 19 November 2019

Published online: 01 January 2020

\section{References}

1. Ritter MA, Davis KE, Meding JB, Pierson JL, Berend ME, Malinzak RA (2011) The effect of alignment and BMI on failure of total knee replacement. J Bone Joint Surg Am 93:1588-1596

2. Parratte S, Pagnano MW, Trousdale RT, Berry DJ (2010) Effect of postoperative mechanical axis alignment on the fifteen-year survival of modern, cemented total knee replacements. J Bone Joint Surg Am 92:21432149

3. Morgan SS, Bonshahi A, Pradhan N, Gregory A, Gambhir A, Porter ML (2008) The influence of postoperative coronal alignment on revision surgery in total knee arthroplasty. Int Orthop 32:639-642

4. Magnussen RA, Weppe F, Demey G, Servien E, Lustig S (2011) Residual varus alignment does not compromise results of TKAs in patients with preoperative varus. Clin Orthop Relat Res 469:3443-3450

5. Liu HX, Shang P, Ying XZ, Zhang Y (2016) Shorter survival rate in varusaligned knees after total knee arthroplasty. Knee Surg Sports Traumatol Arthrosc 24:2663-2671

6. Kim YH, Park JW, Kim JS, Park SD (2014) The relationship between the survival of total knee arthroplasty and postoperative coronal, sagittal and rotational alignment of knee prosthesis. Int Orthop 38:379-385

7. Fang DM, Ritter MA, Davis KE (2009) Coronal alignment in total knee arthroplasty: just how important is it? J Arthroplast 24:39-43

8. Bonner TJ, Eardley WG, Patterson P, Gregg PJ (2011) The effect of postoperative mechanical axis alignment on the survival of primary total knee replacements after a follow-up of 15 years. J Bone Joint Surg (Br) 93:12171222 
9. Cooke TD, Sled EA, Scudamore RA (2007) Frontal plane knee alignment: a call for standardized measurement. J Rheumatol 34:1796-1801

10. Issa SN, Dunlop D, Chang A, Song J, Prasad PV, Guermazi A, Peterfy C, Cahue S, Marshall M, Kapoor D, Hayes K, Sharma L (2007) Full-limb and knee radiography assessments of varus-valgus alignment and their relationship to osteoarthritis disease features by magnetic resonance imaging. Arthritis Rheum 57:398-406

11. Evanich CJ, Tkach TK, von Glinski S, Camargo MP, Hofmann AA (1997) 6- to 10-year experience using countersunk metal-backed patellas. J Arthroplast 12:149-154

12. Scuderi GR, Bourne RB, Noble PC, Benjamin JB, Lonner JH, Scott WN (2012) The new knee society knee scoring system. Clin Orthop Relat Res 470:3-19

13. Matziolis G, Adam J, Perka C (2010) Varus malalignment has no influence on clinical outcome in midterm follow-up after total knee replacement. Arch Orthop Trauma Surg 130:1487-1491

14. Lee BS, Lee SJ, Kim JM, Lee DH, Cha EJ, Bin SI (2011) No impact of severe varus deformity on clinical outcome after posterior stabilized total knee arthroplasty. Knee Surg Sports Traumatol Arthrosc 19:960-966

15. Hinman RS, May RL, Crossley KM (2006) Is there an alternative to the full-leg radiograph for determining knee joint alignment in osteoarthritis? Arthritis Rheum 55:306-313

16. Colebatch AN, Hart DJ, Zhai G, Williams FM, Spector TD, Arden NK (2009) Effective measurement of knee alignment using AP knee radiographs. Knee 16:42-45

17. van Raaij TM, Brouwer RW, Reijman M, Bierma-Zeinstra SM, Verhaar JA (2009) Conventional knee films hamper accurate knee alignment determination in patients with varus osteoarthritis of the knee. Knee 16:109-111

18. Sheehy L, Felson D, Zhang Y, Niu J, Lam YM, Segal N, Lynch J, Cooke TD (2011) Does measurement of the anatomic axis consistently predict hipknee-ankle angle (HKA) for knee alignment studies in osteoarthritis? Analysis of long limb radiographs from the multicenter osteoarthritis (MOST) study. Osteoarthr Cartil 19:58-64

19. Chang CB, Choi JY, Koh IJ, Seo ES, Seong SC, Kim TK (2010) What should be considered in using standard knee radiographs to estimate mechanical alignment of the knee? Osteoarthr Cartil 18:530-538

20. Park A, Stambough JB, Nunley RM, Barrack RL, Nam D (2016) The Inadequacy of Short Knee Radiographs in Evaluating Coronal Alignment After Total Knee Arthroplasty. J Arthroplast 31:878-882

21. Lee SA, Choi SH, Chang MJ (2015) How accurate is anatomic limb alignment in predicting mechanical limb alignment after total knee arthroplasty? BMC Musculoskelet Disord 16:323

22. Abu-Rajab RB, Deakin AH, Kandasami M, McGlynn J, Picard F, Kinninmonth AW (2015) Hip-knee-ankle radiographs are more appropriate for assessment of post-operative mechanical alignment of total knee arthroplasties than standard AP knee radiographs. J Arthroplast 30:695-700

23. Kim JM, Hong SH, Kim JM, Lee BS, Kim DE, Kim KA, Bin SI (2015) Femoral shaft bowing in the coronal plane has more significant effect on the coronal alignment of TKA than proximal or distal variations of femoral shape. Knee Surg Sports Traumatol Arthrosc 23:1936-1942

24. Yau WP, Chiu KY, Tang WM, Ng TP (2007) Coronal bowing of the femur and tibia in Chinese: its incidence and effects on total knee arthroplasty planning. J Orthop Surg (Hong Kong) 15:32-36

25. Ritter MA, Davis KE, Davis P, Farris A, Malinzak RA, Berend ME, Meding JB (2013) Preoperative malalignment increases risk of failure after total knee arthroplasty. J Bone Joint Surg Am 95:126-131

26. Nakano N, Matsumoto T, Hashimura M, Takayama K, Ishida K, Araki D, Matsushita T, Kuroda R, Kurosaka M (2016) Coronal lower limb alignment in normal knees--A radiographic analysis of 797 normal knee subjects. Knee. 23:209-213

27. Kim DK, Seo MC, Song SJ, Kim KI (2015) Are korean patients different from other ethnic groups in total knee arthroplasty? Knee Surg Relat Res 27:199-206

28. Teter KE, Bregman D, Colwell CW Jr (1995) The efficacy of intramedullary femoral alignment in total knee replacement. Clin Orthop Relat Res. 1995; (321):117-21.

29. Lee YS, Lee BK, Lee SH, Park HG, Jun DS, Moon DH (2013) Effect of foot rotation on the mechanical axis and correlation between knee and whole leg radiographs. Knee Surg Sports Traumatol Arthrosc 21:2542-2547

30. Koshino T, Takeyama M, Jiang LS, Yoshida T, Saito T (2002) Underestimation of varus angulation in knees with flexion deformity. Knee 9:275-279

31. Bao Z, Qiao L, Qin J, Xu J, Zhou S, Chen D, Shi D, Dai J, Yao Y, Jiang Q, Xu Z (2017) The assessment of femoral shaft morphology in the sagittal plane in
Chinese patients with osteoarthritis-a radiographic analysis. J Orthop Surg Res 12:127

\section{Publisher's Note}

Springer Nature remains neutral with regard to jurisdictional claims in published maps and institutional affiliations.
Ready to submit your research? Choose BMC and benefit from:

- fast, convenient online submission

- thorough peer review by experienced researchers in your field

- rapid publication on acceptance

- support for research data, including large and complex data types

- gold Open Access which fosters wider collaboration and increased citations

- maximum visibility for your research: over $100 \mathrm{M}$ website views per year

At BMC, research is always in progress.

Learn more biomedcentral.com/submissions 